\title{
Exogenous abscisic acid induces on lipid and flavonoid metabolism of tea plant under drought stress
}

\section{CURRENT STATUS: POSTED}

Research Square

Zhongshuai Gai

Qingdao Agricultural University

Yu Wang

Qingdao Agricultural University

Yiqian Ding

Qingdao Agricultural University

Wenjun Qian

Qingdao Agricultural University

Hui Xie

Qingdao Agricultural University

Litao Sun

Qingdao Agricultural University

Zhongwu Jiang

Yantai University

Qingping $\mathrm{Ma}$

Liaocheng University

Linjun Wang

Fruit tea station of Weihai agricaultural and rural affairs service center

Zhaotang Ding

Qingdao Agricultural University

dzttea@163.comCorresponding Author

ORCiD: https://orcid.org/0000-0002-6814-3038

DOI: 
$10.21203 / \mathrm{rs} .2 .14448 / \mathrm{v} 1$

\section{SUBJECT AREAS}

Plant Physiology and Morphology

KEYWORDS

Camellia sinensis, abscisic acid, metabolomics, transcriptome, drought stress 
Abstract

Background: Abscisic acid (ABA) is an important phytohormone responsible for activating drought resistance, but the regulation mechanism of exogenous $A B A$ on tea plants under drought stress was rarely reported.

Results: The results showed that the exogenous ABA significantly induced the metabolic pathways of tea leaves under drought stress, including the chlorophyll synthesis, photosynthesis, sucrose and starch metabolism, TCA cycle, glycolysis, lipid metabolism and flavonoids biosynthesis. In which, the exogenous ABA could up-regulated the genes related to lipid metabolism and flavonoid biosynthesis, including LPCAT, ALDH, FLS, CHI, DFR, and down-regulated the genes related to lipid metabolism and flavonoid biosynthesis, including FATB, EKI, DGK, PAL, 4CL. The exogenous ABA could also increase the contents of flavone, anthocyanins, flavonol, isoflavone of tea leaves under drought stress, including delphinidin 3-O-glucosidewere, cyanidin 3-O-rutinoside, kaempferitrin, sakuranetin, prunetin, kaempferol, and decrease the contents of glycerophospholipids, glycerolipids and fatty acids of tea leaves under drought stress, including LysoPE 14:0, LysoPE 16:0, LysoPE 18:0, LysoPE 18:1, LysoPC 15:1 and LysoPC 16:0. And there were strong correlations between the genes and metabolites.

Conclusions: The results suggested that the exogenous ABA could alleviate the damages of tea leaves under drought stress through inducing the expressions of the genes and altering the contents of metabolites in response to drought stress. The data also provide a good foundation for further research on the roles of the genes and metabolites in response to ABA.

\section{Background}

Drought is a major abiotic stress for tea plant, it not only limits the productivity of tea plants but also affects the quality of teas [1]. It is essential for us to explore appropriate ways to mitigate the influences of drought stress on tea plants. At present, the researches on the impacts of drought stress on tea plants mainly focused on the mechanisms underlying the stress response, which included morphological, physiological and molecular changes [1-3].

The physiological changes, including the contents of chlorophyll, proline, MDA and the activities of antioxidant enzymes, have been extensively researched in tea plants under drought stress [4, 5]. A large number of drought-inducible genes and proteins in tea plants have also been identified by transcriptome and proteome $[3,6,7]$. A former study in our lab showed that a large numbers of DEGs 
in response to drought stress were mainly enriched in volatile compounds, flavonoids, theanine biosynthesis pathways, and some DEGs were also involved in leaf senescence, such as LOX (lipoxygenase), BGLU6/14/18/41/42 (beta-glucosidases 6/14/18/41/42), BSMT (salicylate/benzoate carboxyl methyltransferase), ADC (arginine decarboxylase), GS (glutamine synthetase), GLS (glutaminase), $\mathrm{CHI}$ (chalcone isomerase), F3'5'H (flavonoid 3',5'-hydroxylase), FLS (flavonol synthase), F3H (flavanone 3-hydroxylase) [1]. Another study in our lab showed that a large numbers of the differentially expressed proteins in response to drought stress were mainly involved in glycolysis/gluconeogenesis, starch and sucrose synthesis, or degradation metabolism, and second metabolism [6]. Recent research in our lab found that many Kub proteins related to ubiquitinmediated proteolysis (e.g., RGLG2, UBC36, UEV1D, RPN10 and PSMC2), catechins biosynthesis (e.g., $\mathrm{PAL}, \mathrm{CHS}, \mathrm{CHI}$ and $\mathrm{F} 3 \mathrm{H}$ ), carbohydrate and amino acid metabolism (e.g., FBPase, FBA and GAD1), were significantly induced by drought stress, suggesting that these Kub proteins might affect the degradation of proteins, the synthesis of catechins, and the accumulation of sucrose, fructose and GABA in tea leaves [7].

Abscisic acid (ABA) is an important phytohormone responsible for activating drought resistance. Drought stress induces ABA biosynthesis, which is the signal that triggers a number of molecular and cellular responses, ultimately resulting in stomatal closure. Several studies indicated that ABA could enhance stress tolerance either as a result of its endogenous concentration or through exogenous application [8, 9]. For instance, the exogenous ABA is beneficial for plants to enhance drought resistance, as it induces stomatal closure, osmotic adjustment, and increases activities of antioxidant enzymes in tomato, spring wheat and Populus $[5,10,11]$. Moreover, the exogenous ABA could improve protein transport, carbon metabolism and expression of resistance proteins to enhance drought tolerance of tea plants [12]. However, to our knowledge, the regulation mechanism of exogenous ABA on genes and metabolites of tea plants under drought stress was rarely reported. In the present study, we analyzed the effects of exogenous ABA on genes and metabolites of tea leaves under drought stress using transcriptome and metabolomics. The study will contribute to further an improved understanding of the molecular mechanism of tea plants in response to drought stress. Results Phenotype and physiological changes of tea leaves under AT and SD To investigate the phenotype changes of tea plants treated with exogenous ABA and drought, their stress phenotypes (displayed visible morphological changes) were photographed. The results showed that a number of wilted and curled leaves were observed under SD (Figure 1). While the wilted and curled leaves were remarkably relieved under AT, indicating that the exogenous ABA partly relieved the damage and maintained the growth of tea plants under drought stress.

To assess the effects of exogenous ABA on physiological characterization of tea leaves, we 
detected several physiological indexes of tea leaves during drought stress. As shown in Table 1, the content of total chlorophyll decreased as the duration of the drought treatment increased under AT and SD, but it was higher under AT than that under SD. The value of maximum quantum yield of PSII ( FV/Fm) also showed similar changes. The content of MDA increased as the duration of the drought treatment increased under AT and SD, but it was lower under AT than that under SD. The results indicated that the exogenous ABA could reduce lipid peroxidation, prevent the degradation of chlorophyll and maintain photosynthesis of tea leaves under drought stress. In addition, the contents of three endogenous hormones and the activities of four antioxidant enzymes were also measured, suggested that the exogenous ABA could affect the changes of endogenous hormones and antioxidant enzymes of tea leaves under drought stress.

Table 1 Physiological indexes of tea leaves during drought stress

\begin{tabular}{lllll}
\hline Parameters & CK & MD & AT & \\
\hline MDAnmol/g & $46.25 \pm 0.88 \mathrm{~b}$ & $43.87 \pm 0.88 \mathrm{~b}$ & $47.54 \pm 0.92 \mathrm{ab}$ & 5 \\
CATU/g & $98.48 \pm 3.29 \mathrm{a}$ & $80.21 \pm 1.75 \mathrm{c}$ & $82.76 \pm 1.72 \mathrm{bc}$ & 8 \\
PODU/g & $105.09 \pm 3.09 \mathrm{a}$ & $110.33 \pm 2.63 \mathrm{a}$ & $89.17 \pm 0.92 \mathrm{~b}$ & 8 \\
APX $(\mu \mathrm{mol} / \mathrm{min} / \mathrm{g})$ & $1.55 \pm 0.05 \mathrm{a}$ & $0.6186 \pm 0.01 \mathrm{c}$ & $0.68 \pm 0.02 \mathrm{c}$ & 0 \\
GR $(\mu \mathrm{mol} / \mathrm{min} / \mathrm{g})$ & $483.26 \pm 2.81 \mathrm{bc}$ & $433.22 \pm 16.81 \mathrm{c}$ & $536.16 \pm 16.96 \mathrm{ab}$ & 5 \\
ABA $(\mu \mathrm{g} / \mathrm{g})$ & $0.4669 \pm 0.0194 \mathrm{~b}$ & $0.533 \pm 0.024 \mathrm{~b}$ & $2.6333 \pm 0.031 \mathrm{a}$ & 0 \\
IAA $(\mu \mathrm{g} / \mathrm{g})$ & $0.6124 \pm 0.0113 \mathrm{~b}$ & $0.8129 \pm 0.0338 \mathrm{a}$ & $0.6303 \pm 0.0158 \mathrm{~b}$ & 0 \\
GA $(\mu \mathrm{g} / \mathrm{g})$ & $0.495 \pm 0.0393 \mathrm{~d}$ & $0.6711 \pm 0.0417 \mathrm{c}$ & $0.9108 \pm 00397 \mathrm{~b}$ & 1 \\
FV/FM & $0.83 \pm 0.21 \mathrm{a}$ & $0.79 \pm 0.19 \mathrm{ab}$ & $0.73 \pm 0.18 \mathrm{~b}$ & \\
Chl contentmg/g & $3.17 \pm 0.33 \mathrm{a}$ & $3.01 \pm 0.27 \mathrm{ab}$ & $2.88 \pm 0.12 \mathrm{ab}$ &
\end{tabular}

The date in the table are represented as the mean \pm standard deviation of three biological replicates, lowercase letters indicated statistical significance-samples not sharing a letter differed significantly according to Duncan test at $\mathrm{P}<0.05$.

The analysis of Transcriptome

To explore the transcript events of exogenous ABA on tea plants under drought, the sample leaves of CK, MD, AT and SD were conducted to RNA-seq analysis. A total of 619,817,958 clean reads were obtained from 12 RNA-Seq libraries. The Q30 percentage were over 92.54\%, and the average GC content was over $44.52 \%$. Overall, the results indicated that the RNA-Seq datasets were robust quality and could be used for further analysis (Supplementary Table S2). 
A total of 2,210 DEGs (1,361 up- and 849 down-regulated) were obtained in AT/MD, 13,007 DEGs (6465 up- and 6542 down-regulated) were obtained in SD/MD, 9,238 DEGs (5006 up- and 4232 down-regulated) were obtained in AT/SD (Figure 2). The results revealed exogenous ABA can effectively relieve the drought stress of tea plants and decrease the expression amounts of response genes to drought.

GO analysis of the above DEGs revealed that the highly enriched terms of the biological process category were 'cellular process', 'metabolic process', 'biological regulation' and 'response to stimulus'. Within the cellular component category, the highly enriched terms were 'cell', 'cell part' and 'organelle'. Within the molecular function category, the highly represented terms were 'binding', 'catalytic activity' and 'transporter activity' (Supplementary Fig S1).

KEGG pathway enrichment analysis revealed that the DEGs in AT/MD were mainly enriched in starch and sucrose metabolism, porphyrin and chlorophyll metabolism, lipid metabolismplantpathogen interaction, plant hormone signal transduction. The DEGs in SD/MD were mainly enriched in photosynthesis, biosynthesis of amino acids, biosynthesis of secondary metabolites, starch and sucrose metabolism. And the DEGs in AT/SD were mainly enriched in starch and sucrose metabolism, lipid metabolismglycolysis and citrate cycle (Supplementary Fig S2).

The effect of exogenous ABA on chlorophyll metabolism and photosynthesis of tea plants under drought stress

To investigate the effect of exogenous ABA on primary metabolism of tea plants under drought stress, we mainly analyzed the expressions of genes related to chlorophyll metabolism, photosynthesis, starch and sucrose metabolism, TCA cycle, glycolysis and lipid metabolism.

For chlorophyll metabolism, the expressions of genes involved in chlorophyll synthesis were down-regulated in SD/MD, but unchanged or slightly down-regulated in AT/MD. For example, CHLS (chlorophyll synthase), PPS (porphobilinogen synthase) and UPD (uroporphyrinogen decarboxylase) were significantly down-regulated in SD/MD, but unchanged in AT/MD. CAO (chlorophyllide a oxygenase) was down-regulated by 3.55-fold in SD/MD, but it was only down-regulated by 2.01-fold in AT/MD. For photosynthesis, the expressions of key genes related to photosynthesis showed similar trends between AT/MD and SD/MD. For example, Rubisco small subunit (CSA018690) were significantly down-regulated by 5.143-fold in SD/MD, but unchanged in AT/MD. Rubisco (CSA035231) was down-regulated by 2.824 -fold in SD/MD, but it was only down-regulated by 1.337 fold in AT/MD (Supplementary Table S3).

The effect of exogenous ABA on energy metabolism and amino acid metabolism of tea plants under drought stress

As for starch and sucrose metabolismthe genes involved in starch synthase were significantly downregulated in SD/MD, but unchanged or slightly down-regulated in AT/MD under drought stress, 
including AGPase (ADP-glucose pyrophosphorylase), SS (starch synthase), GBSS (granule-bound starch synthase), SBE (starch-branching enzyme). While the starch degradation-related genes were up-regulated in SD/MD, but unchanged or slightly up-regulated in AT/MD under drought stress, including AMY ( $\alpha$-amylase) and BAM ( $\beta$-amylase). Similarly, four SPS genes (sucrose-phosphate synthase) involved in sucrose synthesis were down-regulated in SD/MD, but unchanged or slightly down-regulated in AT/MD. While the sucrose degradation-related genes were obviously up-regulated in SD/MD, but unchanged or slightly up-regulated in AT/MD, such as INV (invertase) (Figure 3,

\section{Supplementary Table S3).}

Regarding the glycolysis and TCA cycle, most of genes involved in glycolysis and TCA cycle were significantly up-regulated in SD/MD, but unchanged or slightly up-regulated in AT/MD under drought stress, including HK (hexokinase), PK (pyruvate kinase), PFK (phosphofructokinase), PGAM (phosphoglycerate mutase) in glycolysisACLY (ATP citrate synthase), CS (citrate synthase), Aconitase (aconitate hydratase)IDH (isocitrate dehydrogenase), SCS (succinyl-CoA synthetase) in TCA cycle.

Regarding the amino acid metabolism, the expressions of most genes were significantly upregulated in SD/MD, but unchanged or slightly up-regulated in AT/MD under drought stress, including TAT (tyrosine aminotransferase), ADH (arogenate dehydrogenase), GAD (glutamate decarboxylase), GOGAT (glutamate synthase), ASNS (asparagine synthase).

The effect of exogenous ABA on lipid metabolism of tea plants under drought stress

To investigate the effect of exogenous ABA on lipid metabolism of tea plants, we mainly analyzed the expressions of genes involved in lipid metabolism under drought stress. A total of 81 DEGs related to lipid metabolism were selected, of which 40 DEGs were clearly up-regulated in SD/MD, but unchanged or slightly up-regulated in AT/MD, including DGK (diacylglycerol kinase), FATB (fatty acyl-ACP thioesterase B), EKI (ethanolamine kinase), PLD1/2 (phospholipase D1/2). In addition, 39 DEGs were significantly down-regulated in SD/MD, but unchanged or slightly down-regulated in AT/MD under drought stress, including LOX2S (lipoxygenase), OPR (12-oxophytodienoic acid reductase), PSD (phosphatidylserine decarboxylase), PTDSS (phosphatidylserine synthase). In addition, the LYPLA (lysophospholipase) and LOX1_5 (linoleate 9S-lipoxygenase) were found to be only up-regulated in AT/MD.

The effect of exogenous ABA on phenylpropanoid and flavonoid metabolism of tea plants under drought stress

To investigate the effect of exogenous ABA on phenylpropanoid and flavonoid metabolism of tea plants, we analyzed the expressions of genes involved in phenylpropanoid and flavonoid biosynthesis under drought stress (Figure 4, Supplementary Table S3). The results showed that the vital genes related to phenylpropanoid biosynthesis were highly up-regulated in SD/MD, but unchanged or slightly up-regulated in AT/MD under drought stresssuch as CADcinnamyl-alcohol dehydrogenase $74 C L$ (4- 
coumarate-CoA ligase)F5'H (ferulate-5-hydroxylase), PAL (phenylalanine ammonia-lyase). While the key genes related to flavonoid biosynthesis were significantly down-regulated in SD/MD, but unchanged or slightly down-regulated in AT/MD under drought stress, including F3'H (flavanone-3hydroxylase), CHS (chalcone synthase), CHI (chalcone isomerase), FLS (flavonol synthase), DRF

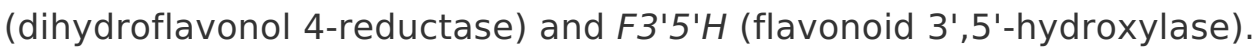

Analysis of drought-responsive transcription factors (TFs)

Transcription factors (TFs) play a key role in the regulation of gene expression under abiotic and biotic stresses in plants. The results showed that more than 50 TFs families were regulated in SD/MD and in AT/MD, implying their important roles in regulating genes involved in drought responses. In which, These TFs, including WRKY, AP2-ERF, NAC, MYB, HSF, bHLH and bZIP, were related to resistance to abiotic and biotic stresses. The result showed that the expressions of the genes encoding these TFs were significantly down/up-regulated in SD/MD, but unchanged or slightly down/up-regulated in AT/MD under drought stress (Supplementary Table S4).

To validate the accuracy and repeatability of transcriptome sequencing dataten DEGs were randomly selected to validate the RNA-seq data by qRT-PCR (Figure 5). The results showed the similar expression patterns between RNA-seq and qRT-PCR, suggesting that the RNA-seq data are reliable.

Metabolic differences of flavonoids and lipid metabolism under AT and SD

To investigate the effects of exogenous ABA on metabolites of tea leaves in response to drought stress, the leaves of CK, MD, AT and SD were conducted to LC-ESI-MS/MS analysis. A total of 65 DEMs (43 up- and 22 down-regulated) were obtained in AT/MD, 90 DEMs (54 up- and 36 down-regulated) were obtained in SD/MD, and 81 DEMs (31 up- and 50 down-regulated) were obtained in AT/SD (Figure 6). Interestingly, the abundances of most flavonoids were markedly increased, while the abundances of most lipid metabolites were markedly decreased in AT/MD compared to those in SD/MD.

For the flavonoids, the most compounds (flavone, flavanone, flavonol, isoflavone and anthocyanins) were obviously increased in AT/MD (Figure 4). Especially, the abundances of sakuranetin in flavone showed 13.15- and 11.06-fold increase in AT/MD and SD/MD, respectively. The levels of prunetin, acacetin, laricitrin and syringetin were only increased in AT/MD. The levels of apigenin O-malonylhexoside, delphinidin 3-O-glucoside and cyanidin 3-O-rutinoside were significantly decreased in SD/MD. In addition, the levels of kaempferol, kaempferitrin, 4-methylcatechol, cyanin and fustin were higher in AT/MD than that in SD/MD. $=3$

For the lipid metabolites (Table 2), 38 metabolites involved in fatty acids, glycerolipids and glycerophospholipids metabolisms were markedly changed under drought stress. Especially, LysoPS 22:6 showed higher abundance with 11.57- and 10-fold increase in SD/MD and AT/MD, respectively. In 
addition, the levels of LysoPE 14:0, LysoPE 16:0, LysoPE 18:0, LysoPE 18:1, LysoPE 18:2 (2n isomer), LysoPC 15:1, LysoPC 16:0 and LysoPC 17:0 increased significantly in SD/MD. The levels of PC 16:1/14:1 and 13-HOTrE were only increased in AT/MD. In addition, 13 metabolites were only increase in SD/MD, such as, LysoPC 18:0 (2n isomer), LysoPC 18:2 (2n isomer) and MAG (18:1) isomer2. Interaction network analysis between genes and metabolites Gene-metabolite interaction networks could be used to help understand functional relationship and to aid in identifying new regulatory elements [13]. Here, Pearson correlation tests were carried out between differentially expressed genes and metabolites related to flavonoids and lipid metabolism.

For phenylpropanoid and flavonoid biosynthesis, both 113 DEGs and 22 DEMs related to phenylpropanoid and flavonoids biosynthesis were carried out pearson correlation analysis. The result showed that 43 DEGs had strong positive and negative correlation coeffcient values (R2 $>0.8$ or $<$ -0.8 and P-value < 0.05) with 12 metabolites (Supplementary Table S5). For example, there was significantly positive correlation between the gene expression (CCR, DFR, CHI3, PER42) and metabolite abundances (gallocatechin, apigenin O-malonylhexoside, delphinidin 3-O-glucoside), but there was significantly negative correlation between the gene expression (CAD) and metabolite abundances (apigenin O-malonylhexoside, gallocatechin, dihydromyricetin and mirtillin).

For lipid metabolism, both 81 DEGs and 38 DEMs related to lipid metabolisms were carried out pearson correlation analysis, the result showed that 56 DEGs had strong positive and negative correlation coeffcient values (R2> 0.8 or $<-0.8$ and P-value $<0.05$ ) with 34 metabolites (Figure 7,

Supplementary Table S6). Further analysis indicated that there was significantly positive correlation between the expressions of eight key genes and abundances of most lipid metabolite, including DGK1/2 (diacylglycerol kinase1/2), FATB (fatty acyl-ACP thioesterase B), EKI (ethanolamine kinase), ACAA1 (acetyl-CoA acyltransferase 1), GPX(glutathione peroxidase), EPHX2 (soluble epoxide hydrolase), LPT1(lysophospholipid acyltransferase1). There was significantly negative correlation between the expressions of two genes and abundances of most lipid metabolite, including LPCAT (lysophosphatidylcholine acyltransferase) and ALDH (aldehyde dehydrogenase).

Discussion

ABA is a pivotal hormone in plant responses to biotic and abiotic stress, playing a key role in adapting metabolism and gene expression to help plant cope with stress conditions [14].Previous research in tea plants showed that the exogenous ABA could improve protein transport, carbon metabolism and enhance the expressions of resistance proteins (Hsp70) to enhance drought tolerance of tea plants [12]. In the present study, to gain further insight into the effects of exogenous ABA on genes and metabolites of tea plants under drought stress, we integrated transcriptome and metabolomics analyses to explore the regulation mechanism. The results showed that exogenous ABA could induce the expressions of the genes related to energy metabolism, lipid metabolism and flavonoid 
biosynthesis to promote energy storages and the balance of primary metabolism, maintain membrane integrity, produce more flavonoids and derivatives in response to drought stress.

Exogenous ABA increased the expression of genes involved in chlorophyll synthesis and photosynthesis

Photosynthesis is sensitive to drought stress, which reduces the net photosynthetic rate and photosynthetic carbon metabolism or even impairs the primary photosynthetic machinery [15]. Previous research demonstrated that the expressions of $p s b A$ and $p s b D$ involved in photosynthesis were repressed to a low level of expression in response to drought stress, resulting in the decrease of chlorophyll contents and the inhibition of PSII activity [16]. Several studies in different plants species showed that the exogenous ABA could significantly ameliorate the effects of drought-induced chlorophyll reduction and alleviate the damage of drought to PSII [5, 15, 17]. However, in tea plants, the effect of exogenous ABA on genes related to chlorophyll synthesis and photosynthesis was rarely reported. In the present study, transcriptome analysis showed that the exogenous ABA significantly induced increase in the expressions of genes involved in chlorophyll synthesis (CHLS, PPS, CAO) and photosynthesis (Rubisco, RbcS) of tea leaves compared with severe drought, suggesting that the exogenous ABA could alleviate the stress injury to chlorophyll synthesis and photosynthesis of tea plants under drought stress. Similar to the expression patterns of genes, physiological results showed that the exogenous ABA significantly increased the chlorophyll contents, raised the value of $F v / F m$ and alleviated the accumulation of MDA compared with severe drought. The results demonstrated that the exogenous ABA could induce increase in the expressions of genes involved in chlorophyll synthesis and photosynthesis, and maintain chlorophyll synthesis and photosynthesis of tea plants under drought stress.

Exogenous ABA promoted energy storages and maintained the balance of primary metabolism Starch is a key molecule in mediating plant responses to abiotic stresses, such as water deficit. Under drought stress conditions, plants generally remobilize starch to provide energy and carbon at times when photosynthesis is potentially limited [18]. The presence of sufficient sucrose can serve as the important energy source for the cells, and they act as an essential osmoprotectant to protect biomembranes and proteins against abiotic stress [19]. Previous study in tea plants showed that the expressions of the genes related to starch synthesis (AGPase, SS) were inhibited and the expressions of the genes related to starch degradation ( $A M Y, B A M)$ were induced, whereas the expressions of the genes related to sucrose synthesis (UDPGase, SPS) and degradation (INVS) were highly induced under drought stress. [1, 2]. However, in tea plants, the effect of exogenous ABA on genes involved in starch and sucrose metabolism was rarely reported.

In the present study, transcriptome analysis showed that the exogenous ABA significantly induced increase in the expressions of the genes related to starch and sucrose synthesis (AGPase, 
SS, SPS) and decrease in the expressions of the genes related to starch and sucrose degradation ( $A M Y, B A M, I N V s)$ of tea leaves compared with severe drought (Figure $\mathbf{3}$ ), suggesting that the exogenous ABA might promote a shift in metabolism towards a storage metabolism in tea leaves under drought stress. In addition, the exogenous ABA significantly induced decrease in the expressions of genes involved in TCA cycle (including ACLY, CS and SCS), glycolysis (including $H K, P K$ and $G A P D H$ ) and amino acid metabolism (including $A D H, G A D$ and GOGAT) compared with severe drought. Metabolomic results showed that the exogenous ABA significantly increased the contents of the ketoglutaric acid, succinic acid and citric acid compared with severe drought. The results indicated that the exogenous ABA possible played the important role in promoting the energy storages and maintaining the balance of primary metabolism of tea plants under drought stress. Exogenous ABA affected the expressions of genes related to lipid metabolism in tea leaves under drought stress

Lipids provide not only the energy for metabolic processes but also the structural basis for cell membranes [20]. Altered lipid biosynthesis and specific fatty acid reduced the impairment of cell membranes under drought stress [21]. Several studies showed that lipid metabolism in different plants was modified by drought stress [22-25]. In the moss Atrichum androgynum, the contents of phosphoglyceride and monogalactosyldiacylglycerol (MGDG) were reduced significantly, but the content of triacylglycerols (TAGs) was increased significantly under drought stress [25]. In peanut, the contents of phospholipids and galactolipids were decreased significantly under drought stress[24]. In addition, several studies showed that ABA could regulate lipid metabolism in different plant species $[26,27]$. For example, the exogenous ABA on mungbean leaves induced a slight increase in the content of phospholipids and a decrease in the content of free fatty acids, whereas monogalactosyldiacylglycerol (MGDG) content was significantly reduced in N-leaves after application of $A B A[27]$. However, in tea plants, the effect of exogenous ABA on genes and metabolites related to lipid metabolism in response to drought stress was rarely reported.

In this work, transcriptome analysis showed that the exogenous ABA significantly induced the expressions of genes related to lipid biosynthesis and degradation of tea leaves under drought stress. The expressions of the FATB, EKI, ACAA1, DGK and GPX were significantly down-regulated and the expressions of the LPCAT and ALDH were significantly up-regulated by exogenous ABA. However, to our knowledge, the association between the expressions of these genes and drought resistance of tea plants was not reported. Metabolomic analysis showed that the exogenous ABA significantly altered the abundances of most lipid metabolites of tea leaves under drought stress. The abundances of LysoPe 14:0, LysoPe 16:0, LysoPe 18:0, LysoPE 18:1, LysoPC 15:1 and LysoPC 16:0 were significantly reduced and the abundances of PC 16:1/14:1, 13-HOTrE and MAG (18:3) isomer4 were slightly increased by exogenous ABA. To date, the association between the changes of these lipid 
metabolites and drought resistance of tea plants was also rarely reported. While the correlation analysis of genes and metabolites showed that eight genes,FATB, DGK1/7, EKI, ACAA1, GPX, LPT1, EPHX2, were significantly positive correlation with most lipid metabolites (including LysoPE 14:0, LysoPE 16:0, LysoPe 18:0, LysoPE 18:1, LysoPC 15:1 and LysoPC 16:0 ), and two genes, LPCAT and ALDH, were significantly negative correlation with most lipid metabolites (Figure 7, Supplementary Table 56), suggesting that the exogenous ABA could induce the expressions of genes related to lipid metabolism for tea leaves to cause the reduction of lipid metabolites under drought stress. Previous study in vigna unguiculata leaf demonstrated a role of $A B A$ in the cell membrane protection against water stress by preventing drought-induced membrane lipid degradation [28]. Similar result was observed in the moss Atrichum androgynum that the exogenous ABA might reduce the membrane damage by diminishing the lipid changes [25]. Previous search showed that the changes in lipid metabolism was correlated with alterations in membrane integrity under water stress [29, 30]. In the present study of tea leaves, the results showed that exogenous ABA could mediate the expression of genes involved in lipid metabolism and affect the changes in lipid metabolism for maintaining membrane integrity and stability under drought stress. The interaction of ABA signaling with lipid signaling in tea plants under drought stress will be further elucidated in our future studies.

Exogenous $A B A$ increased the expressions of genes related to flavonoid biosynthesis in tea leaves under drought stress

Flavonoids are ubiquitous secondary metabolites with a vast array of biological functions, including defense against biotic and abiotic stresses [31]. The flavonoid biosynthesis of tea plants was affected by various environmental conditions, such as drought stress [32]. In the study, we analyzed the effects of exogenous ABA on genes and metabolites related to flavonoid metabolism of tea leaves under drought stress using transcriptome and metabolomics.

Previous researches on tea plants showed that the expressions of the genes related to flavonoid biosynthesis were firstly decreased and subsequently increased in response to drought stress, such as CHS, DFR, LAR and ANR, and the expressions of FLS and FNS were continuously upregulated in response to drought stress [3]. The expressions of the genes related to flavonoid biosynthesis, such as PAL, C4H, 4CL, CHS and DFR, were significantly induced by drought [1]. However, in tea plants, the effect of exogenous ABA on genes related to flavonoids biosynthetic in response to drought stress was rarely reported. In the present study, transcriptome analysis revealed that the exogenous ABA significantly induced the expressions of genes related to flavonoid biosynthesis of tea leaves under drought stress. The expressions of $P A L, 4 C L, F 5^{\prime} H$ were highly downregulated and the expressions of $C H I, D R F, F 3^{\prime} H$ and $F L S$ were significantly up-regulated by exogenous ABA.

Several researches on tea plants showed that the amounts of total polyphenols and catechins, 
including GC, EGC, C, EC, EGCG, GCG, ECG, significantly decreased in response to drought stress [3, 33-35], but the content of total flavonoids significantly increased in response to drought stress [3]. However, in tea plants, there was limited information about the effect of exogenous ABA on flavonoid metabolites in response to drought stress. In the present study, metabolomics analysis revealed that the exogenous ABA significantly affected the abundances of flavonoid metabolites of tea leaves under drought stress. The abundances of flavone, anthocyanins, flavonol, isoflavone classes, including apigenin O-malonylhexoside, delphinidin 3-O-glucosidewere, cyanidin 3-O-rutinoside, kaempferitrin, sakuranetin, prunetin, kaempferol, fustin, ancyanidin 3,5-0-diglucoside, significantly increased and the abundances of flavonol, flavone and flavone C-glycosides, including dihydromyricetin, acacetin Oacetyl hexoside, acacetin O-glucuronic acid, 6-C-hexosyl-hesperetin O-hexosidewere slightly decreased by exogenous ABA.

We also performed the correlation analysis between the genes and flavonoid metabolites of tea leaves under exogenous ABA. The results showed that the expressions of CHI (CSA026735) and DFR (CSA003949) were significantly positive correlation with apigenin O-malonylhexoside, delphinidin 3-O-glucoside, gallocatechin and dihydromyricetin, while the expression of 4CL (CSA007753) was significantly negative correlation with apigenin O-malonylhexoside and delphinidin 3-O-glucoside, suggesting that the exogenous ABA could induce the expressions of genes involved in flavonoid biosynthesis, and hence affected the biosynthesis of flavonoids and derivatives of tea leaves under drought stress.

Moreover, previous research showed that inhibition of lipid synthesis redirects the carbon flux into flavonoid metabolism [36]. Recently, CHS-mediated flavonoids were proven to repress embryonic fatty acid biosynthesis in the Arabidopsis seeds, implying a negative correlation between flavonoids and fatty acids biosynthesis in plants [37]. Our results showed that the exogenous ABA drastically decreased the abundances of lipid metabolites and increased the abundances of most flavonoids compared with severe drought. Therefore, another possible explanation was that the exogenous ABA induced the expression of genes involved in starch and sucrose metabolism to promote the accumulation of more carbon sources, and a large amount of carbon sources flux into flavonoid metabolism to increase the abundances of flavonoids and derivatives, so as to improve drought resistance of tea plants. Further studies are required to confirm such assumptions.

\section{Conclusion}

In this study, we analyzed the roles of exogenous ABA on genes and metabolites of tea leaves under drought stress using transcriptome and metabolomics. The study demonstrated that exogenous ABA significantly reduce the damage of drought to tea plants, maintain the balance of primary metabolism, promote energy storages and the formation of flavonoids and derivatives to enhance drought tolerance of tea plants under drought stress. This study will be helpful for us to understand 
the mechanism of resilience to abiotic stress in tea plant and provide novel insights into enhancing drought tolerance of tea plants in the future.

\section{Methods}

Plant materials and stress treatments

Tea plant cultivar, 'QN3' (Camellia sinensis cv. QN3) is an improved cultivar bred by the Tea Research Institute, Qingdao Agricultural University[38-40] . The two-year-old tea seedling were cultured in growth chamber for 2 weeks with photoperiod $\left(12 \mathrm{~h}\right.$ light at $25^{\circ} \mathrm{C}$ and $12 \mathrm{~h}$ dark at $20^{\circ} \mathrm{C}, 75 \%$ of humidity) and a light intensity of 18, 000 Lux. There were four groups in this experiment: wellwatered plants (CK); based on previous studies and physiological response of tea plants under drought stress [3, 7], we chose the samples that had been drought treated for $24 \mathrm{~h}$ as mild drought (MD); after collection of samples, one group was immediately sprayed with $100 \mathrm{~mL}$ of ABA solution (concentration $50 \mathrm{mg} \mathrm{L}^{-1}$ ) (AT), the other was immediately sprayed with equal parts of distilled water (SD), the two groups were continuous drought and other conditions were unchanged in these treatments. after 53 hours, the samples were collected when the leaf displayed visible morphological changes. Three independent biological replicates were performed at each sampling time, and each replicate contained 10 mature leaves (the second leaf at the top). The samples were immediately frozen in liquid nitrogen and stored at $-80^{\circ} \mathrm{C}$ for further analysis.

Measurement of total chlorophylls, Fv/Fm and MDA

Leaf chlorophyll was extracted with ethanol according to previous studies [39]. Third leaf was sampled to measure maximum quantum yield of PSII according to previous studies [1]. The contents of malondialdehyde (MDA) in the leaves were measured by the thiobarbituric acid (TBA) method according to previous studies [12]. All experiments were carried out with at least three independent repetitions.

RNA-Seq analysis

For RNA-seq analysis, the total RNA of each sample was extracted as described by Wang et al [41]. RNA concentration and integrity of the total RNA were measured using NanoDrop 2000 Spectrophotometer (IMPLEN, Westlake Village, CA, USA) and Agilent 2100 Bioanalyzer (Agilent Technologies, CA, USA), respectively. Subsequently, the library preparations were sequenced on the Illumina HiSeq platform to generate raw data. After sequencing, the clean reads were obtained by removing reads containing adaptors, more than $5 \%$ unknown bases and low-quality reads ( $>20 \%$ of the bases with a quality score of $\leq 10$ ). Gene function was annotated based on the following databases: NCBI non-redundant protein sequences (NR), Clusters of Orthologous (KOG/COG), Gene Ontology (GO), manually annotated and reviewed protein sequence database (Swiss-Prot), Kyoto Encyclopedia of Genes and Genomes (KEGG). Gene expression levels were represented using fragments per kilobase of transcript per million fragments mapped (FPKM) method. The differentially 
expressed genes (DEGs) were recruited based on False Discovery Rate (FDR) $<0.05$ and $\mid \log _{2}$ Fold Change| $\geq 1$. All DEGs were analyzed by GO enrichment using GOseq (1.10.0) [42] and KEGG enrichment using KOBAS software [43].

Quantitative real-time RT-PCR (qRT-PCR) analysis

Total RNA was extracted by using a plant RNA extraction kit (Tiangen, Beijing, China). First-strand cDNA was reverse transcribed according to the user manual of PrimeScript ${ }^{\mathrm{TM}} \mathrm{RT}$ reagent kit (TaKaRa), and the qRT-PCR program was performed by using a Light Cycler 480 instrument (Roche). The genes were selected for RT-qPCR with specific primers designed by Primer Premier 5 software (Supplementary Table S1). The qRT-PCR reagents were the following: a total of $20 \mu \mathrm{L}$ of reaction mixture, which included $10 \mu$ I SYBR Green PCR Master Mix (Roche), $1 \mu$ of each primer, $2 \mu$ l cDNA and $6 \mu$ distilled water. The qPCR program was performed as follows: $95^{\circ} \mathrm{C}, 10 \mathrm{~min} ; 95^{\circ} \mathrm{C}, 10 \mathrm{~s}$ and $60^{\circ} \mathrm{C}$, $15 \mathrm{~s}$ for 45 cycles; then a melting curve. GAPDH was used as reference gene for quantifying the expression levels of the target genes according to the method of $2_{-}^{-\Delta \Delta \mathrm{Ct}}$ [44]. The quantitative analysis of each RNA sample was repeated at least three times, and the representative data are expressed as the mean values \pm standard error $(n=3)$.

Metabolite profiling analysis

Sample preparation, metabolite extraction and analysis were carried out as described in previous research $[45,46]$. In brief, the $100 \mathrm{mg}$ freeze-dried samples were extracted using $1.0 \mathrm{~mL} 70 \%$ aqueous methanol (containing $0.1 \mathrm{mg} / \mathrm{L}$ lidocaine). Subsequently, 10,000 g centrifugation for $10 \mathrm{~min}$ at $4^{\circ} \mathrm{C}$, then the extracts were absorbed and filtered before LC-MS analysis. A quality-control sample was prepared by equal blending of all samples; during the assay, the quality control sample was run every 10 injections to monitor the stability of the analytical conditions. The extracted samples $(2 \mu \mathrm{l})$ were analyzed using a HPLC system (Shim-pack UFLC SHIMADZU CBM 30A) equipped with Waters

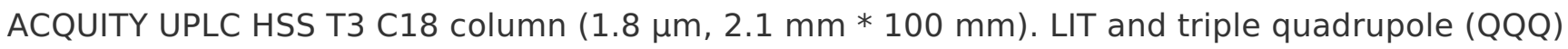
scans were acquired on a QTRAP-MS equipped with an ESI Turbo lon-Spray interface under AB Sciex QTRAP 4500 System, a positive ion mode and controlled by Analyst 1.6.1 software (AB Sciex). The solvent system, gradient program and ESI source operation parameters were carried out as described by previous research [47].

The qualitative analysis of primary and secondary MS data was performed by searching the internal database using a self-compiled database MWDB (MetWare Biological Science and Technology Co., Ltd. Wuhan, China), Data pre-processing and metabolites identification were performed by the standard metabolic procedures, including comparing the $\mathrm{m} / \mathrm{z}$ values, RT, and the fragmentation patterns with the standards. The variable importance of the projection (VIP) score of the application (O) PLS model was used to filter the best differentiated metabolites between treatments. Metabolites with significant 
differences in content were set with thresholds of variable importance in projection (VIP) $\geq 1$ and fold change $\geq 2$ or $\leq 0.5$.

Statistical analysis

All statistical analyses were performed with SPSS 20.0 (Windows; SPSS Inc., Chicago, IL, USA) and significance tests were determined by Duncan's test and ANOVA. $P<0.05$ was considered the significant difference. The relationships visualization was performed by Cytoscape software (version 3.3.0, USA). Integrative analysis of metabolome and transcriptome was carried out using $\mathrm{R}$ software (version 3.2.4, USA).

\section{Declarations}

Ethics approval and consent to participate

Not applicable.

Consent for publication

Not applicable.

Availability of data and materials

All the data supporting the findings of the study is contained within the manuscript and its additional information files.

Competing interests

The authors declare that they have no competing interests.

Funding

This research was subsidized by the Significant Application Projects of Agriculture Technology Innovation in Shandong Province, the Breeding Project of Shandong Province (2017LZN014), the Technology System of Modern Agricultural Industry in Shandong Province (SDAIT-19-01), the Special Foundation for Distinguished Taishan Scholar of Shandong Province (No.ts201712057), and the National Natural Science Foundation of China (No. 31800588). These funding bodies played no role in the design of the study and collection, analysis, and interpretation of data and in writing the manuscript.

Authors' contributions

ZD conceived the idea. ZD and ZG designed the study. ZG, HX, LS, YD and LW performed the experiments. ZG, WQ and QM analyzed the data with the input from YW. ZD, and ZG wrote the manuscript. ZG, ZD, YW, and ZJ critically read the manuscript. All authors were involved in critically reading and editing the manuscript and gave the final approval of the version to be published. All authors read and approved the final manuscript.

Acknowledgements

We thank MetWare Biological Science and Technology Co., Ltd. (Wuhan, China) for the technical 
assistances.

\section{References}

1. Zheng C, Wang Y, Ding Z, Zhao L: Global Transcriptional Analysis Reveals the Complex Relationship between Tea Quality, Leaf Senescence and the Responses to Cold-Drought Combined Stress in Camellia sinensis. Frontiers in Plant Science 2016, 7:1858.

2. Liu SC, Jin JQ, Ma JQ, Yao MZ, Ma CL, Li CF, Ding ZT, Chen L: Transcriptomic Analysis of Tea Plant Responding to Drought Stress and Recovery. Plos One 2016, 11(1):e0147306.

3. Wang W, Xin H, Wang M, Ma Q, Wang L, Kaleri NA, Wang Y, Li X: Transcriptomic Analysis Reveals the Molecular Mechanisms of Drought-Stress-Induced Decreases in Camellia sinensis Leaf Quality. Front Plant Sci 2016, 7(795):385.

4. Upadhyaya H, Panda SK: Responses ofCamellia sinensisto Drought and Rehydration. Biologia Plantarum 2004, 48(4):597-600.

5. Du Y, Wang Z, Fan J, Turner N, He J, Wang T, Li F: Exogenous abscisic acid reduces water loss and improves antioxidant defence, desiccation tolerance and transpiration efficiency in two spring wheat cultivars subjected to a soil water deficit. Functional Plant Biology Fpb 2013, 40(5):494-506.

6. Wang Y, Fan K, Wang J, Ding ZT, Wang H, Bi CH, Zhang YW, Sun HW: Proteomic analysis of Camellia sinensis (L.) reveals a synergistic network in the response to drought stress and recovery. Journal of Plant Physiology 2017, 219:91-99.

7. Xie H, Wang Y, Ding Y, Qiu C, Sun L, Gai Z, Gu H, Ding Z: Global Ubiquitome Profiling Revealed the Roles of Ubiquitinated Proteins in Metabolic Pathways 
of Tea Leaves in Responding to Drought Stress. Scientific Reports 2019 , $9(1): 4286$.

8. Haisel D, Pospí?ilová J, Synková H, Schnablová R, Ba?ková P: Effects of abscisic acid or benzyladenine on pigment contents, chlorophyll fluorescence, and chloroplast ultrastructure during water stress and after rehydration. Photosynthetica 2006, 44(4):606-614.

9. Trouverie J, Thevenot C, Rocher JP, Sotta B, Prioul JL: The role of abscisic acid in the response of a specific vacuolar invertase to water stress in the adult maize leaf. Journal of Experimental Botany 2003, 54(390):2177-2186.

10. Aroca R, Alguacil MDM, Vernieri P, Ruiz-Lozano JM: Plant Responses to Drought Stress and Exogenous ABA Application are Modulated Differently by Mycorrhization in Tomato and an ABA-deficient Mutant ( Sitiens ). Microbial Ecology 2008, 56(4):704-719.

11. Chunyang LI, Yin C, Liu S: Different responses of two contrasting Populus davidiana populations to exogenous abscisic acid application. Environmental \& Experimental Botany 2004, 51(3):237-246.

12. Zhou L, Xu H, Mischke S, Meinhardt LW, Zhang D, Zhu X, Li X, Fang W: Exogenous abscisic acid significantly affects proteome in tea plant (Camellia sinensis) exposed to drought stress. Horticulture Research 2014, 1:14029.

13. Wu ZG, Jiang W, Chen SL, Mantri N, Tao ZM, Jiang CX: Insights from the Cold Transcriptome and Metabolome ofDendrobium officinale: Global Reprogramming of Metabolic and Gene Regulation Networks during Cold Acclimation. Frontiers in Plant Science 2016, 7(e0121658).

14. Antoni R, Gonzalez-Guzman M, Rodriguez L, Peirats-Llobet M, Rodriguez PL: PYRABACTIN RESISTANCE1-LIKE8 plays an important role for the regulation 
of abscisic acid signaling in root. Plant Physiology 2013, 161(2):931-941.

15. Teng K, Li J, Liu L, Han Y, Du Y, Zhang J, Sun H, Zhao Q: Exogenous ABA induces drought tolerance in upland rice: the role of chloroplast and ABA biosynthesis-related gene expression on photosystem II during PEG stress. Acta Physiologiae Plantarum 2014, 36(8):2219-2227.

16. Hui-Guo, Duan, Yuan, Wen-Juan, De-Hui, Dong-Hong, Qing, Hou-Guo, Liang, Hong-Hui: Effects of Exogenous Spermidine on Photosystem II of Wheat Seedlings Under Water Stress. Journal of Integrative Plant Biology 2006, 48(8):920-927.

17. Zhao PF: Effect of Exogenous Abscisic Acid on psbA Expression at Grain Filling Stage in Two Wheat Cultivars Under Drought Stress. Acta Agronomica Sinica 2011, 37(8):1372-1377.

18. Thalmann M, Santelia D: Starch as a determinant of plant fitness under abiotic stress. New Phytol 2017, 214(3):943-951.

19. Cao YY, Yang M-T, Li X, Zhou Z-Q, Wang X-J, Bai J-G: Exogenous sucrose increases chilling tolerance in cucumber seedlings by modulating antioxidant enzyme activity and regulating proline and soluble sugar contents. Scientia Horticulturae 2014, 179:67-77.

20. Yozo O, Kazuki S: Roles of lipids as signaling molecules and mitigators during stress response in plants. Plant Journal 2014, 79(4):584-596.

21. Deng S, Ma J, Zhang L, Chen F, Sang Z, Jia Z, Ma L: De novo transcriptome sequencing and gene expression profiling of Magnolia wufengensis in response to cold stress. BMC Plant Biology 2019, 19(1):321.

22. Liljenberg CS: The effects of water deficit stress on plant membrane lipids. Progress in Lipid Research 1992, 31(3):335-343.

23. Repellin A, Thi ATP, Tashakorie A, Sahsah Y, Daniel C, Zuily-Fodil Y: Leaf membrane 
lipids and drought tolerance in young coconut palms (Cocos nucifera L.). European Journal of Agronomy 1997, 6(96):25-33.

24. Lauriano JA, Lidon FC, Carvalho CA, Campos PS, Matos MDC: Drought Effects on Membrane Lipids and Photosynthetic Activity in Different Peanut Cultivars. Photosynthetica 2000, 38(1):7-12.

25. Guschina IA, Harwood JL, Smith M, Beckett RP: Abscisic Acid Modifies the Changes in Lipids Brought about by Water Stress in the Moss Atrichum androgynum. New Phytol 2002, 156(2):255-264.

26. Ritchie S, ., Gilroy S, . Abscisic acid stimulation of phospholipase D in the barley aleurone is G-protein-mediated and localized to the plasma membrane. Plant Physiology 2000, 124(2):693-702.

27. Aghofack-Nguemezi J, Christmann A, Frosch S, Trémoliéres A, Wagner E: Contrasting photo- and thermoperiod-induced changes in abscisic acid and lipid contents in leaves of mungbean seedlings. Physiologia Plantarum 2010, 83(3):346-352.

28. Campos PS, Thi ATP: Effects of an abscisic acid pretreatment on membrane leakage and lipid composition of Vigna unguiculata leaf discs subjected to osmotic stress. Plant Science 1997, 130(1):11-18.

29. Taranto PA, Keenan TW, Potts M, . Rehydration induces rapid onset of lipid biosynthesis in desiccated Nostoc commune (Cyanobacteria). Biochim Biophys Acta 1993, 1168(2):228-237.

30. Platt KA, Oliver MJ, Thomson WW: Membranes and organelles of dehydrated Selaginella and Tortula retain their normal configuration and structural integrity. Protoplasma 1994, 178(1-2):57-65.

31. Broun P: Transcriptional control of flavonoid biosynthesis: a complex network of conserved regulators involved in multiple aspects of differentiation in 
Arabidopsis. Current Opinion in Plant Biology 2005, 8(3):272-279.

32. Jaleel CA, Sankar B, Murali PV, Gomathinayagam M, Lakshmanan GM, Panneerselvam R: Water deficit stress effects on reactive oxygen metabolism in Catharanthus roseus; impacts on ajmalicine accumulation. Colloids \& Surfaces B Biointerfaces 2008, 62(1):105-111.

33. Munivenkatappa N, Sarikonda S, Rajagopal R, Balakrishnan R: Variations in quality constituents of green tea leaves in response to drought stress under south Indian condition. Scientia Horticulturae 2018, 233:359-369.

34. Chen XH, Zhuang CG, He YF, Wang L, Han GQ, Chen C, He HQ: Photosynthesis, yield, and chemical composition of Tieguanyin tea plants ( Camellia sinensis (L.) O. Kuntze) in response to irrigation treatments. Agricultural Water Management 2010, 97(3):419-425.

35. Jeyaramraja PR, Pius PK, Kumar RR, Jayakumar D: Soil moisture stress-induced alterations in bioconstituents determining tea quality. Journal of the Science of Food \& Agriculture 2003, 83(12):1187-1191.

36. Leonard E, Lim KH, Saw PN, Koffas MAG: Engineering central metabolic pathways for high-level flavonoid production in Escherichia coli. Applied \& Environmental Microbiology 2007, 73(12):3877-3886.

37. Xuan L, Zhang C, Yan T, Wu D, Hussain N, Li Z, Chen M, Pan J, Jiang L:

TRANSPARENT TESTA 4-mediated flavonoids negatively affect embryonic fatty acid biosynthesis in Arabidopsis. Plant Cell and Environment 2018, $41(12): 2773-2790$.

38. Jiang J, Gai Z, Wang Y, Fan K, Sun L, Wang H, Ding Z: Comprehensive proteome analyses of lysine acetylation in tea leaves by sensing nitrogen nutrition. BMC Genomics 2018, 19(1):840. 
39. Gai ZS, Wang Y, Jiang JT, Xie H, Ding ZT, Ding SB, Wang H: The Quality Evaluation of Tea (Camellia sinensis) Varieties Based on the Metabolomics. Hortscience 2019, 54(3):409-413.

40. Sun JH, Qiu C, Qian WJ, Wang Y, Sun LT, Li YS, Ding ZT: Ammonium triggered the response mechanism of lysine crotonylome in tea plants. BMC Genomics 2019, 20.

41. Wang L, Cao H, Qian W, Yao L, Hao X, Li N, Yang Y, Wang X: Identification of a novel bZIP transcription factor in Camellia sinensis as a negative regulator of freezing tolerance in transgenic arabidopsis. Ann Bot 2017, 119(7):11951209.

42. Young MD, Wakefield MJ, Smyth GK, Oshlack A: Gene ontology analysis for RNAseq: accounting for selection bias. Genome Biology 2010, 11(2):R14-R14.

43. Mao X, Tao CJGO, Wei L: Automated genome annotation and pathway identification using the KEGG Orthology (KO) as a controlled vocabulary. Bioinformatics 2005, 21(19):3787-3793.

44. Livak KJ, Schmittgen TD: Analysis of relative gene expression data using realtime quantitative PCR and the 2(-Delta Delta C(T)) Method. Methods 2001, $25(4): 402-408$.

45. Wang Z, Cui Y, Vainstein A, Chen S, Ma H: Regulation of Fig (Ficus caricaL.) Fruit Color: Metabolomic and Transcriptomic Analyses of the Flavonoid Biosynthetic Pathway. Frontiers in Plant Science 2017, 8:1990-.

46. Shen J, Wang Y, Chen C, Ding Z, Hu J, Zheng C, Li Y: Metabolite profiling of tea ( Camellia sinensis L.) leaves in winter. Scientia Horticulturae 2015, 192:1-9.

47. Chen W, Gong L, Guo Z, Wang W, Zhang H, Liu X, Yu S, Xiong L, Luo J: A Novel Integrated Method for Large-Scale Detection, Identification, and 
Quantification of Widely Targeted Metabolites: Application in the Study of

Rice Metabolomics. Molecular Plant 2013, 6(6):1769-1780.

Figures

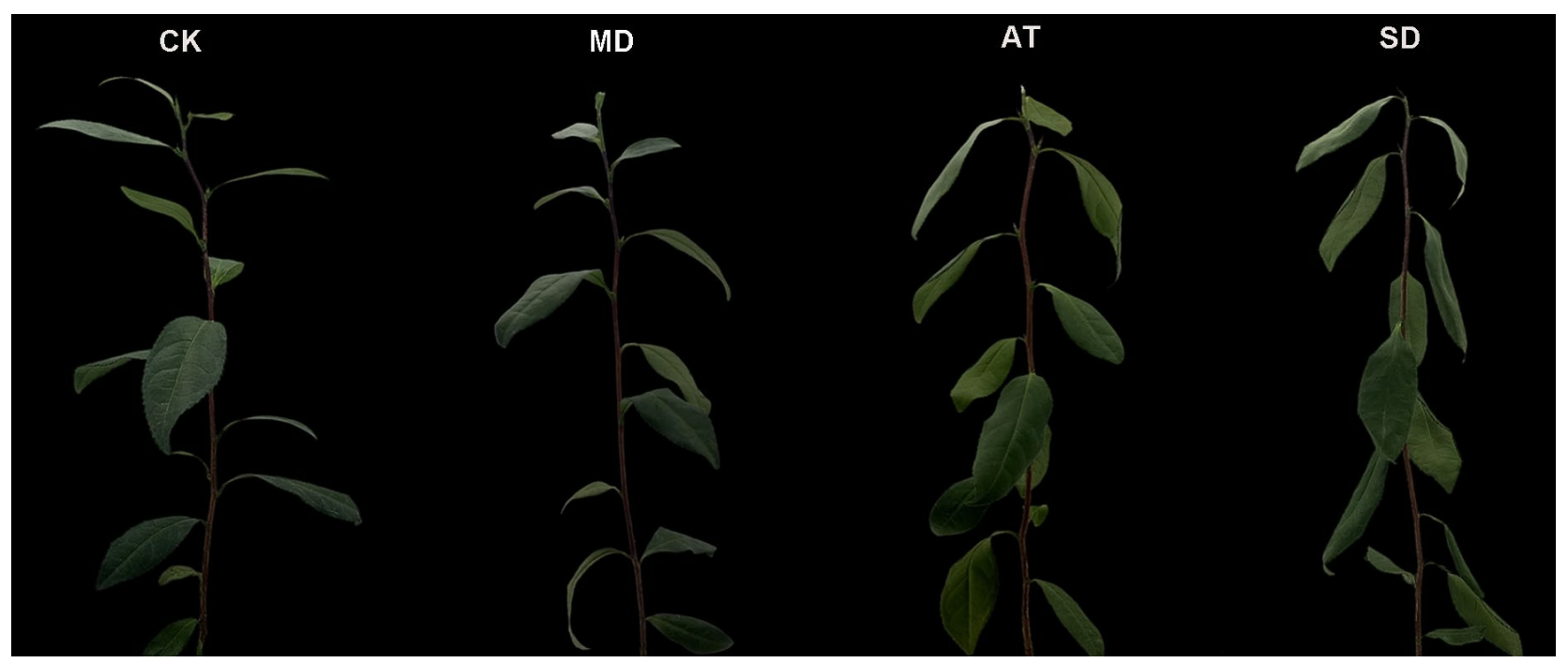

Figure 1

The phenotype changes of tea leaves under CK, MD, AT and SD. 


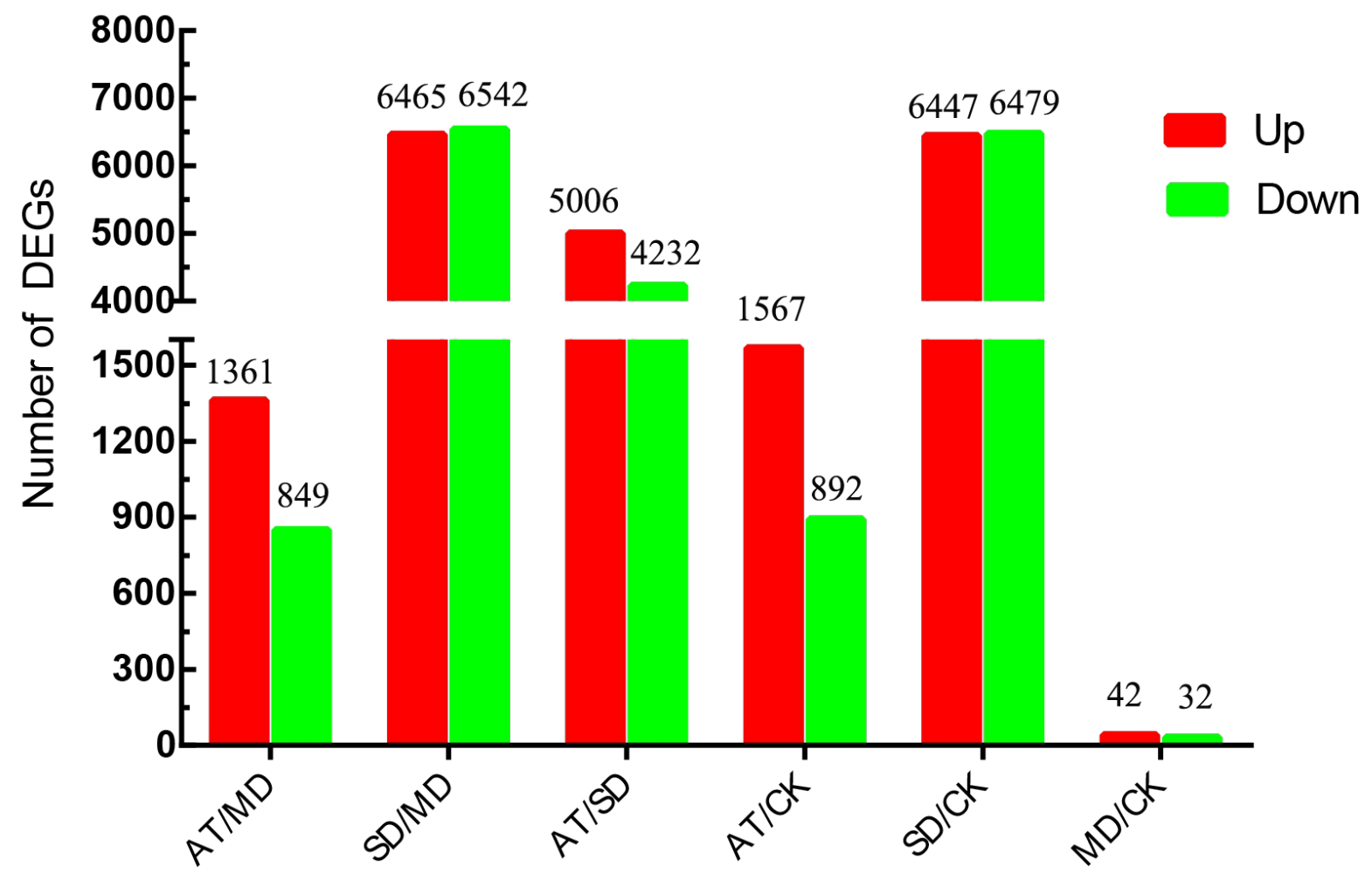

Figure 2

Statistics of the differentially expressed genes (DEGs) between AT/MD, SD/MD and AT/SD. 


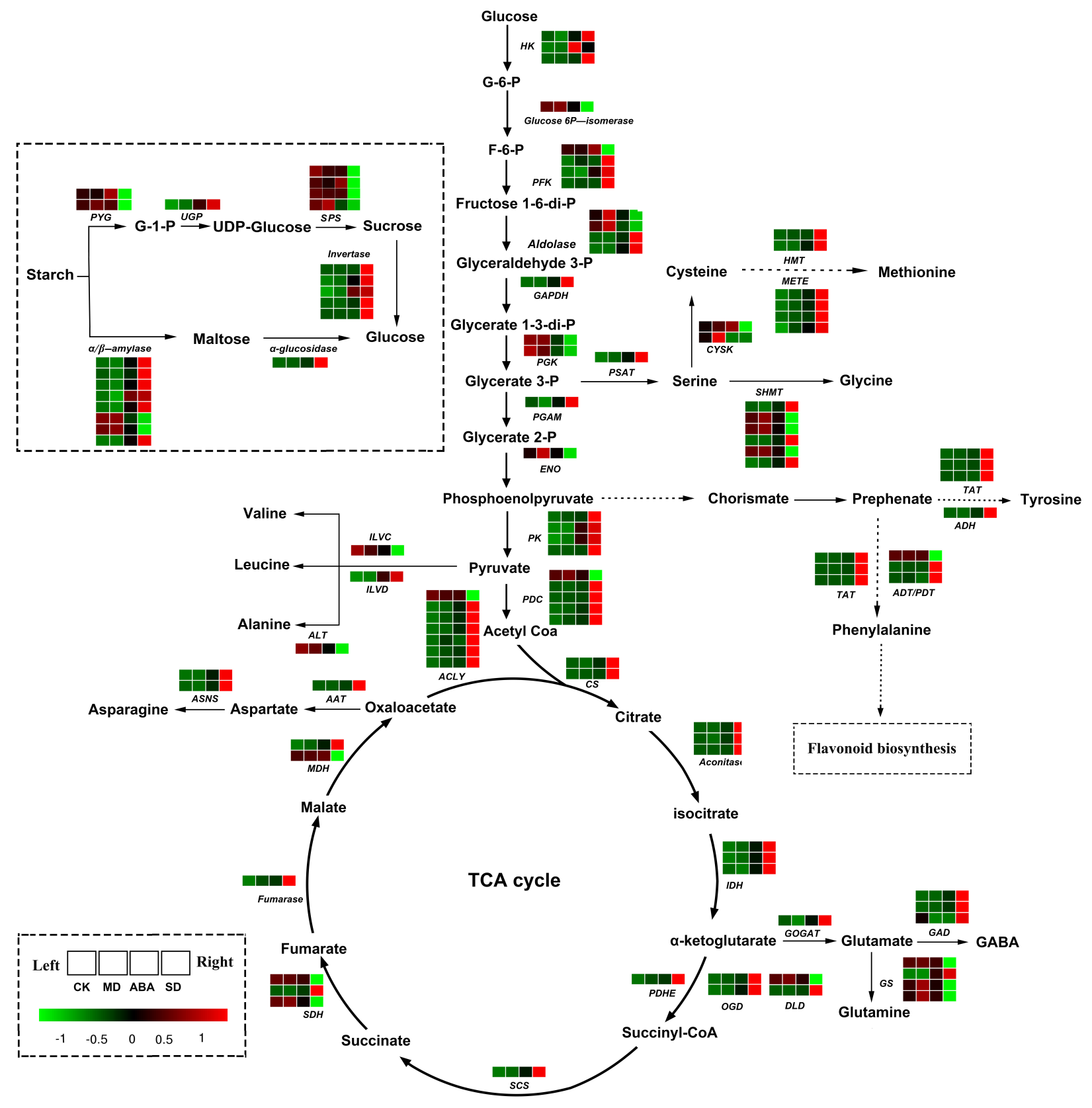

Figure 3

The response of genes related to starch and sucrose metabolism, glycolysis, TCA cycle and amino acids pathway under exogenous $A B A$ and severe drought (SD). 


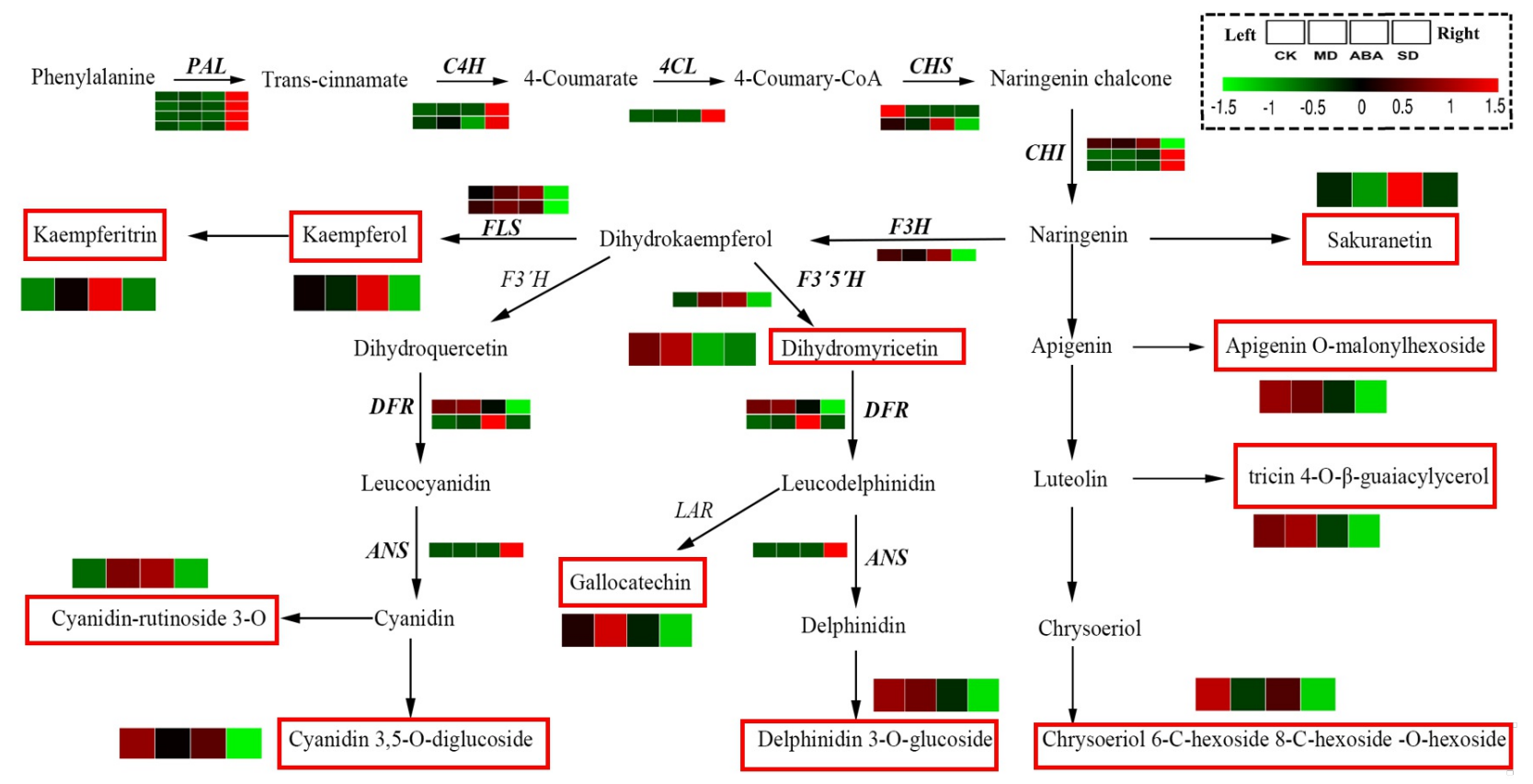

Figure 4

The expressions of genes related to phenylpropanoid and flavonoid metabolism in response to exogenous $A B A$ and severe drought (SD).

EKI-CSA030599

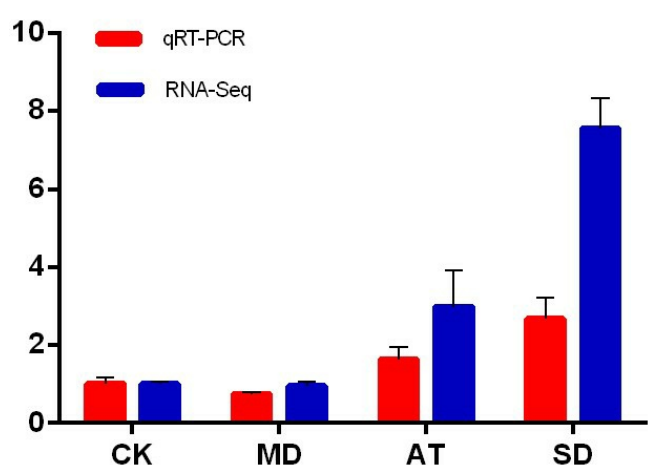

LPLAT1-CSA032782

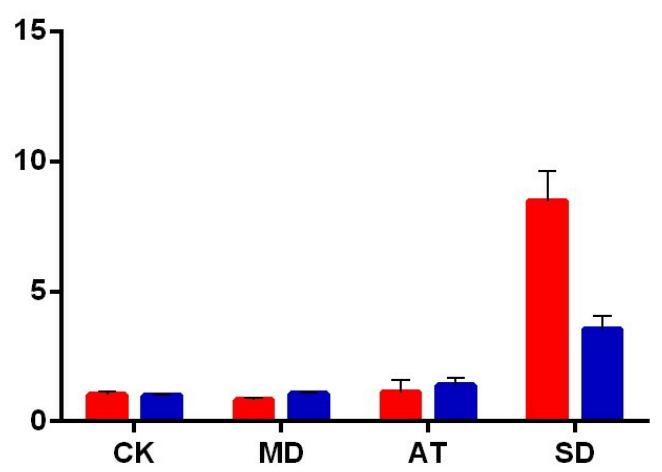

LPPH-CSA011597

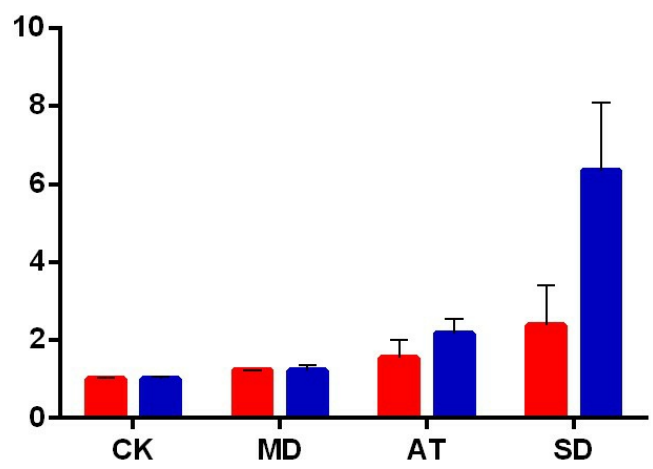

FATB-CSA000990

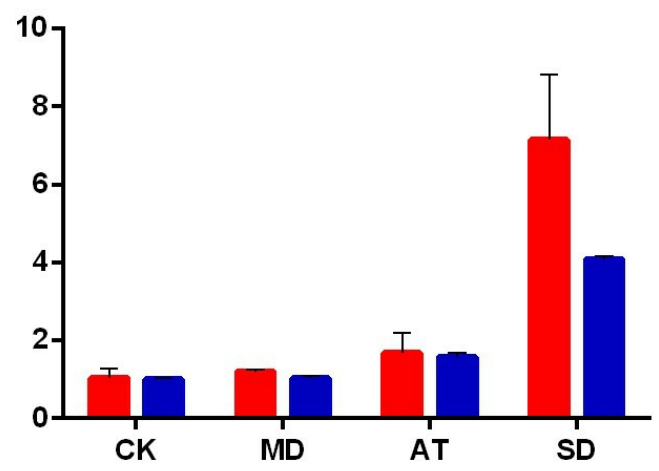


REF1-CSA016635

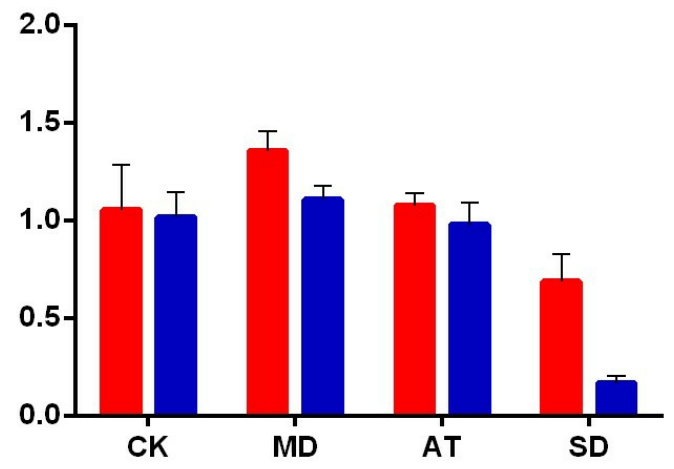

DFR-CSA003949

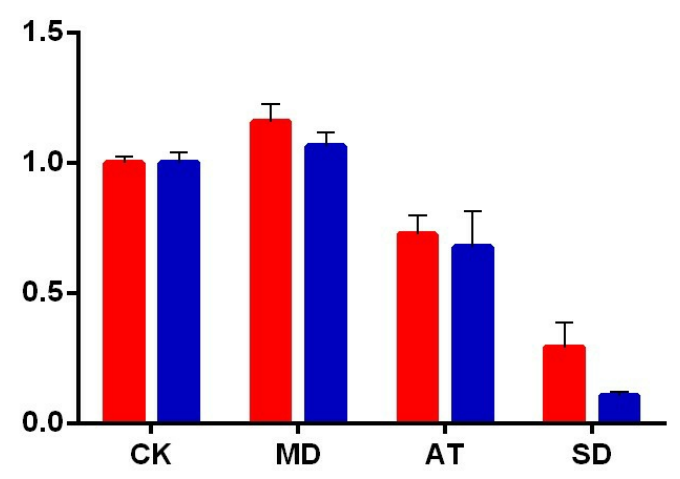

CCR-CSA000931

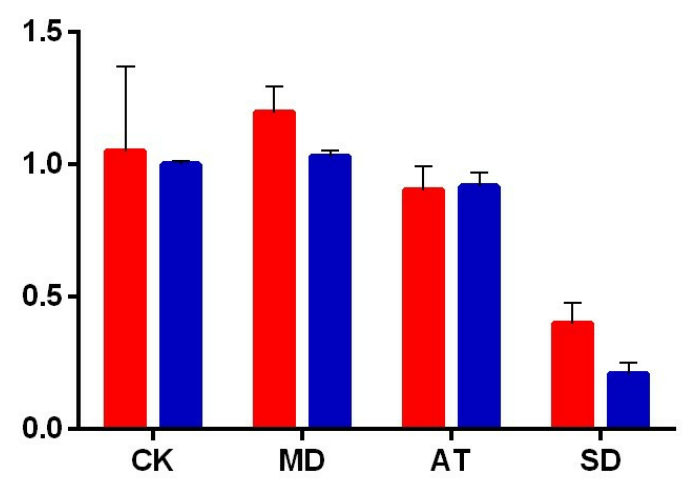

CHI-CSA026735

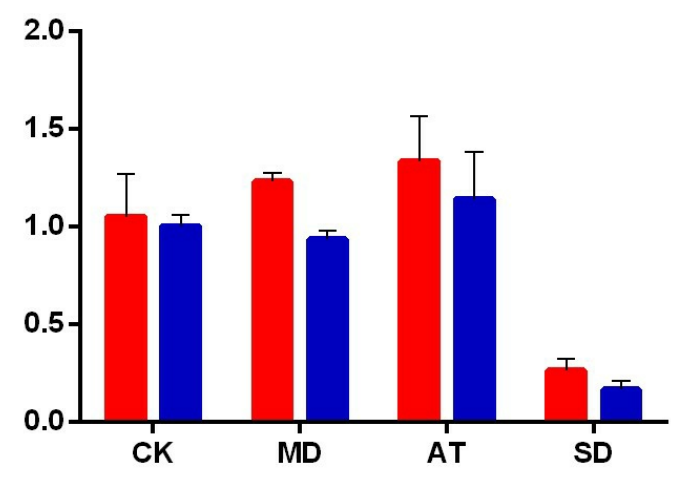

HK-CSA020560

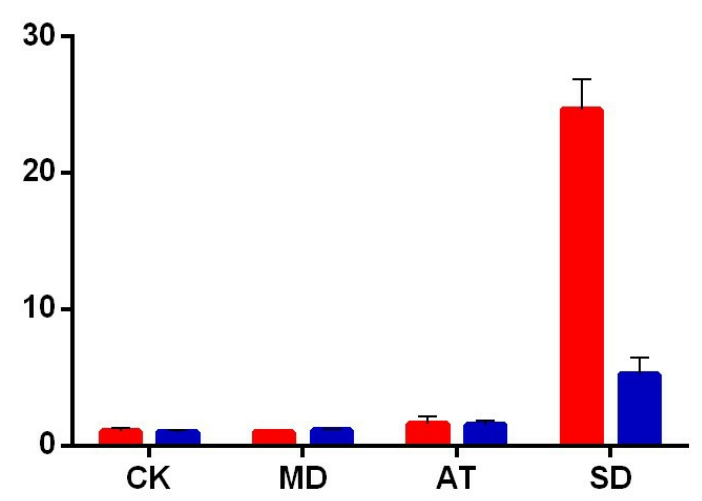

CHI-CSA033689

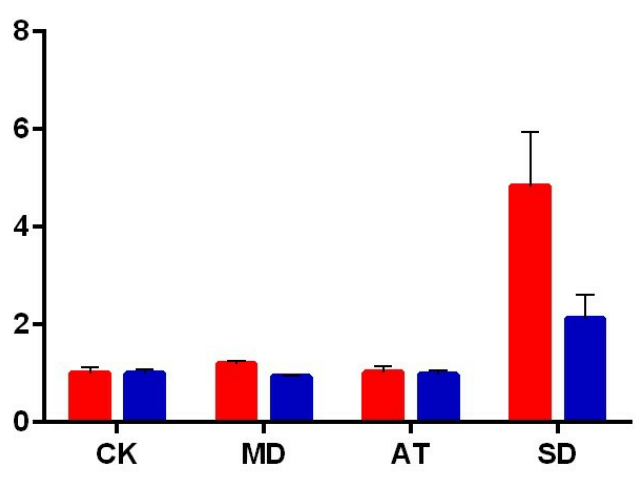

Figure 5

qRT-PCR analysis of a set of DEGs. The expression at CK was set as 1 , and the relative expression level was calculated for several genes. 


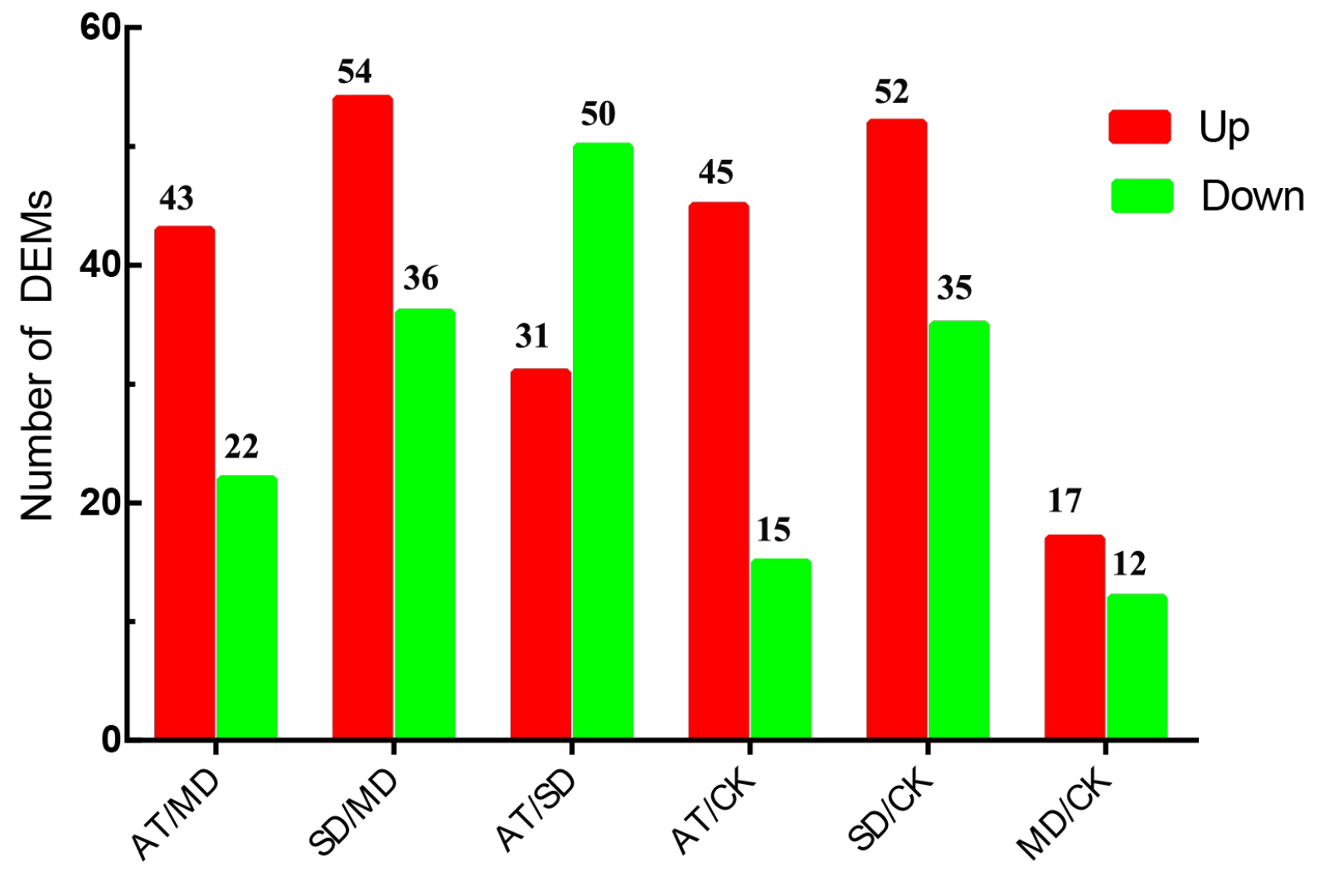

Figure 6

Statistics of the differentially expressed metabolites (DEMs) between AT/MD, SD/MD and AT/SD. 


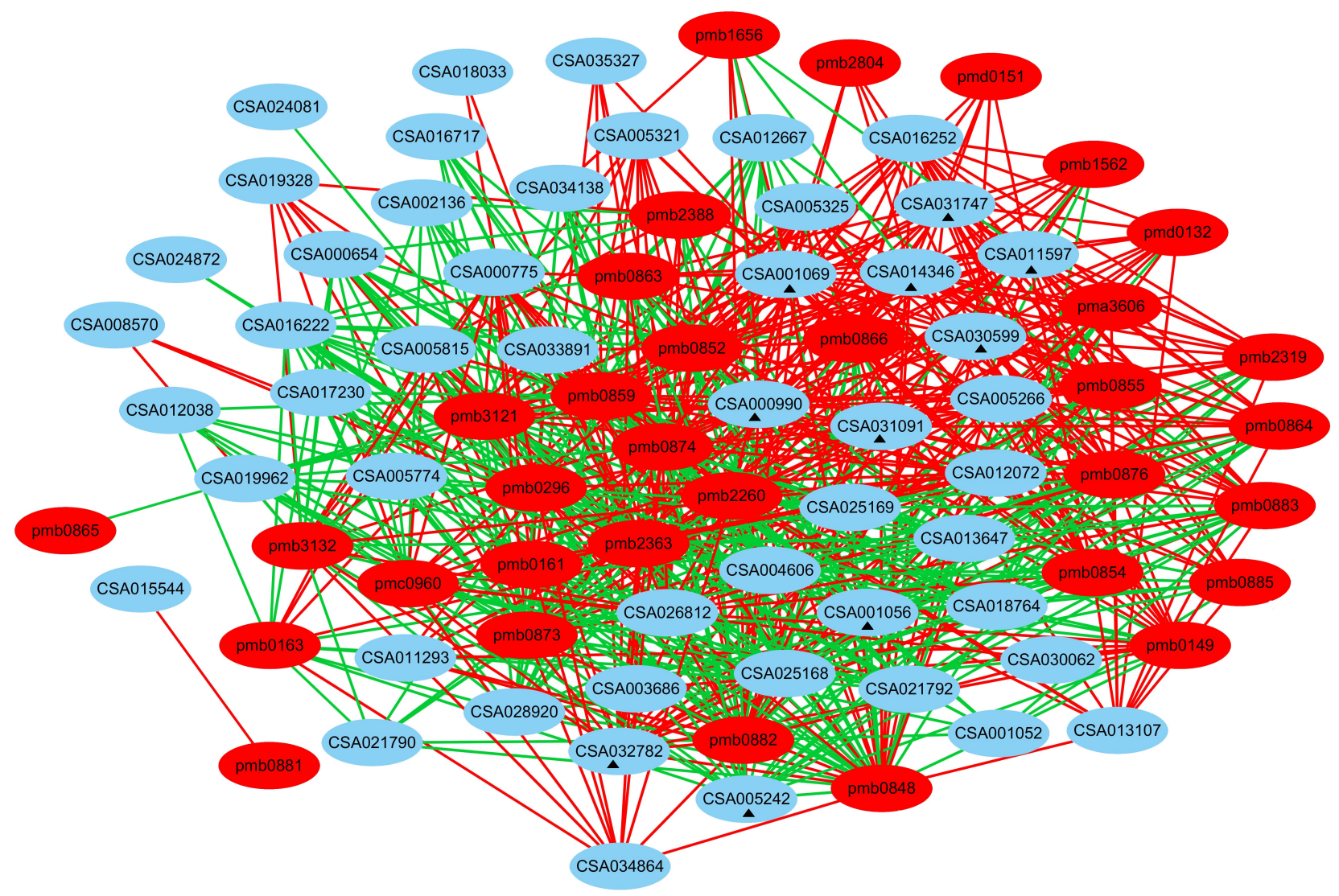

Figure 7

The connection network between genes and metabolites related to lipid metabolism.

\section{Supplementary Files}

This is a list of supplementary files associated with this preprint. Click to download.

Table S4.xIsx

Supplementary Table S1.docx

Table S3.xIsx

Fig S1.png

Table S6.xIsx

Supplementary Table S2.docx

Fig S2.png

Table S5.xlsx 